EUROPEAN JOURNAL OF PURE AND APPLIED MATHEMATICS

Vol. 13, No. 3, 2020, 663-673

ISSN 1307-5543 - www.ejpam.com

Published by New York Business Global

\title{
Some Localization of the Zeros of the Third Derivative of a Complex Polynomial in the Disks or Generalized Cardioid Interiors
}

\author{
Todor Stoyanov Stoyanov
}

Department of Mathematics, University of Economics, bul. Knyaz Boris I 7r, Varna 9002, Bulgaria

\begin{abstract}
In this paper, we localize the zeros of the third derivatives of a complex polynomial in some sets. These sets are relevant to the first, second and third derivative of the polynomial, and they are respectively disks and cardioid interiors or generalized cardioid interiors. Here, for the first time we consider generalized cardioids, as the areas of zeros.
\end{abstract}

2020 Mathematics Subject Classifications: 30D20

Key Words and Phrases: Zeros, Complex Polynomial, Disks, Cardioid Interiorities

\section{Introduction}

The localization of the zeros of the complex polynomials is very important area of the mathematics. The impossibility to find the zeros of any polynomials using the coefficients makes every statement here very significant. There exist many conjectures which are not proved, like Sendov's conjecture, Obreshkoff's conjecture. The assertions localize the zeros of the derivative of the any complex polynomial in some areas. Here we present some new results about the zeros of the derivative of the complex polynomials. Theorem 1 could be seen in [3]. Theorem 3, Theorem 4 and Theorem 5 we can see in [4]. Their results could be applied for the localization of the zeros of the derivative of the polynomials- these are Theorem 3 and Theorem 4. In Theorem 5 we localize the zeros of the second derivative of the complex polynomial. Theorem 6 and the Corollary could be used everywhere in the fields of mathematics, independently of their application here in Theorem 7 . Theorem 7 appears the main result of the article.

For the first time here, we consider a generalized cardioid. We see that the roots of polynomial must belong to the generalized cardioid interiorities, created by the zeros of the given polynomial. Many of these results could be applied for the solving of the unproved conjectures. Especially that could be seen in [1]. Other possibilities are $[2,5]$ and [6].

DOI: https://doi.org/10.29020/nybg.ejpam.v13i3.3736

Email address: todstoyanov@yahoo.com (T. S. Stoyanov) 


\section{Preliminaries}

We note:

$D(a, r)=\{z \in C:|z-a|<r\}$ is the open disk.

$\overline{D(a, r)}=\{z \in C:|z-a| \leq r\}$ is the closed disk.

$C(a, r)$ - the open cardioid interior - how to define it: after translation t, $t(a)=r \in R$ and then rotation with angle $\varphi=-\arg a ; a \in C$. Then coordinates must satisfy

$$
\left(x^{2}+y^{2}-2 r x\right)^{2}<4 r^{2}\left(x^{2}+y^{2}\right) .
$$

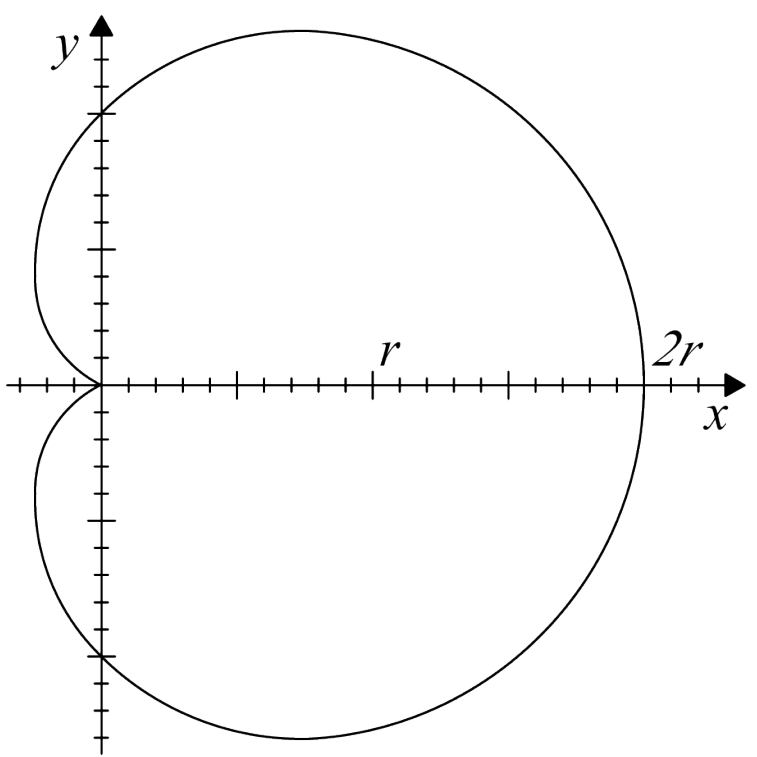

Figure 1:

$\overline{C(a, r)}$ - the closed cardioid interior.

$C_{q}(a, r)$ - the open generalized cardioid interior - how define it: after translation $t, t(a)=r \in R$ and then a rotation with angle $\varphi=-\arg a ; a \in R$. The coordinates must satisfy

$$
\left(x^{2}+y^{2}-2 r x\right)<4 q^{2} r^{2}\left(x^{2}+y^{2}\right)
$$

$\overline{C_{q}(a, r)}$ - the closed generalized cardioid interior.

Sendov's conjecture: Let us put for $n \geq 2, p(z)=\prod_{k=1}^{n}\left(z-z_{k}\right)$, where $z_{k} \in$ $\overline{D(0,1)}, k=1,2, \ldots, n$. Then $p^{\prime}(z)$ has at least one zero in each of the disks $\overline{D\left(z_{k}, 1\right)}, k=$ $1,2, \ldots n$.

\section{Related Results}

Theorem 1. Let the zeros $z_{k}, k=1,2, \ldots, n$ of a polynomial $p(z) \in C[z]$ satisfy $z_{k} \in$ $\overline{D(0,1)}$. Then the zeros $z$ of the polynomial $q(z)=\gamma p(z)+z p^{\prime}(z)$, where $\operatorname{Re} \gamma \geq-\frac{n}{2}$, 
satisfy $z \in \overline{D(0,1)}$.

Proof. Let $z$ be such that $q(z)=0$ and $p(z) \neq 0$. Then

$$
\frac{q(z)}{p(z)}=\gamma+\frac{z}{z-z_{1}}+\cdots+\frac{z}{z-z_{k}}=0
$$

Hence

$$
\begin{aligned}
& \frac{q(z)}{p(z)}=\gamma+\frac{\frac{z}{2}-\frac{z_{1}}{2}+\frac{z}{2}+\frac{z_{1}}{2}}{z-z_{1}}+\cdots+\frac{\frac{z}{2}-\frac{z_{n}}{2}+\frac{z}{2}+\frac{z_{n}}{2}}{z-z_{k}}=0 . \\
& \frac{q(z)}{p(z)}=\gamma+\frac{n}{2}+\frac{1}{2}\left[\frac{\left(z+z_{1}\right)\left(\bar{z}-\overline{z_{1}}\right)}{\left|z-z_{1}\right|^{2}}+\cdots+\frac{\left(z+z_{n}\right)\left(\bar{z}-\overline{z_{n}}\right)}{\left|z-z_{n}\right|^{2}}\right]=0 .
\end{aligned}
$$

Therefore

$$
\operatorname{Re} \frac{q(z)}{p(z)}=\operatorname{Re} \gamma+\frac{n}{2}+\frac{1}{2}\left[\frac{|z|^{2}-\left|z_{1}\right|^{2}}{\left|z-z_{1}\right|^{2}}+\cdots+\frac{|z|^{2}-\left|z_{n}\right|^{2}}{\left|z-z_{n}\right|^{2}}\right]=0 .
$$

If we assume $z \notin \overline{D(0,1)}$, then we obtain $\operatorname{Re} \gamma>0$, when is impossible.

Theorem 2. If all the zeros $z_{k}, k=1,2, \ldots n$; of a polynomial $p(z) \in C[z]$ satisfy $z_{k} \in$ $\overline{D(0,1)}$ and $a$ is a zero of $p(z)$ of modulus 1 , then the derivative $p^{\prime}(z)$ has at least one zero in $\overline{D\left(\frac{a}{2}, \frac{1}{2}\right)}$.

Proof. Let $p(z)=(z-a) q(z)$. If we denote by $z_{1}, z_{2}, \ldots, z_{n-1}$ the zeros of $q(z)$ and by $w_{1}, w_{2}, \ldots w_{n-1}$ those of $p^{\prime}(z)$, then in the non-trivial case $q(a) \neq 0$ we obtain

$$
\begin{aligned}
\sum_{k=1}^{n-1} \operatorname{Re} \frac{a}{a-w_{k}} & =\operatorname{Re} \frac{a p^{\prime \prime}(a)}{p^{\prime}(a)}==2 \operatorname{Re} \frac{q^{\prime}(a)}{q(a)} \\
& =2 \sum_{k=1}^{n-1} \operatorname{Re} \frac{a}{a-z_{k}} \geq 2 \frac{n-1}{2}=n-1 .
\end{aligned}
$$

Here we essentially, that

$$
\begin{aligned}
p^{\prime}(z) & =(z-a) q^{\prime}(z)+q(z), \\
p^{\prime \prime}(z) & =(z-a) q^{\prime \prime}(z)+2 q(z),
\end{aligned}
$$

and $|a|=1$.

Hence $\operatorname{Re} \frac{a}{a-w_{k}} \geq 1$ for some $k,(1 \leq k \leq n-1)$. That means

$$
R e \frac{\frac{a}{2}-\frac{w_{k}}{2}+\frac{a}{2}+\frac{w_{k}}{2}}{a-w_{k}}=\operatorname{Re}\left(\frac{1}{2}+\frac{1}{2} \frac{a+w_{k}}{a-w_{k}}\right)
$$




$$
=\operatorname{Re}\left[\frac{1}{2}+\frac{1}{2} \frac{\left(a+w_{k}\right)\left(\bar{a}-\overline{w_{k}}\right)}{\left|a-w_{k}\right|^{2}}\right]=\frac{1}{2}+\frac{1}{2} \frac{|a|^{2}-\left|w_{k}\right|^{2}}{\left|a-w_{k}\right|^{2}} \geq 1,
$$

i.e. $\left|a-w_{k}\right|^{2}+\left|w_{k}\right|^{2} \leq|a|^{2}$ which confirms that $w_{k} \in \overline{D\left(\frac{a}{2}, \frac{1}{2}\right)}$.

Theorem 3. If all the zeros of a polynomial $p(z) \in C[z]$ are $z_{k}, k=1,2, \ldots, n$. Then for each zero $w$ of the derivative $p^{\prime}(z)$ exists some $k_{0} \in N, 1 \leq k_{0} \leq n$, such that $w \in \overline{D\left(\frac{z_{k_{0}}}{2}, \frac{\left|z_{k_{0}}\right|}{2}\right)}$.

Proof. Let $w \in C$ be such that $p^{\prime}(w)=0$. We except the trivial case $p(w)=0$, which confirms the assertion. Then

$$
\begin{aligned}
w \frac{p^{\prime}(w)}{p(w)} & =\frac{w}{w-z_{1}}+\frac{w}{w-z_{2}}+\cdots+\frac{w}{w-z_{n}} \\
& =\frac{\frac{w}{2}-\frac{z_{1}}{2}+\frac{w}{2}+\frac{z_{1}}{2}}{w-z_{1}}+\frac{\frac{w}{2}-\frac{z_{2}}{2}+\frac{w}{2}+\frac{z_{2}}{2}}{w-z_{2}}+\cdots+\frac{\frac{w}{2}-\frac{z_{n}}{2}+\frac{w}{2}+\frac{z_{n}}{2}}{w-z_{k}} \\
& =\frac{n}{2}+\frac{1}{2}\left[\frac{\left(w+z_{1}\right)\left(\bar{w}-\overline{z_{1}}\right)}{\left|w-z_{1}\right|^{2}}+\cdots+\frac{\left(w+z_{n}\right)\left(\bar{w}-\overline{z_{n}}\right)}{\left|w-z_{n}\right|^{2}}\right]=0 .
\end{aligned}
$$

Therefore

$$
\operatorname{Re} w \frac{p^{\prime}(w)}{p(w)}=\frac{n}{2}+\frac{1}{2}\left[\frac{|w|^{2}-\left|z_{1}\right|^{2}}{\left|w-z_{1}\right|^{2}}+\cdots+\frac{|w|^{2}-\left|z_{n}\right|^{2}}{\left|w-z_{n}\right|^{2}}\right]=0 .
$$

We put

$$
\alpha_{k}=\frac{|w|^{2}-\left|z_{n}\right|^{2}}{\left|w-z_{n}\right|^{2}}, k=1,2, \ldots n .
$$

Then $\alpha_{1}+\alpha_{2}+\cdots+\alpha_{n}=-n$. Consequently there exists $k_{0} \in N, \quad 1 \leq k_{0} \leq n$ such that $\alpha_{k_{0}} \leq-1$. It means that $|w|^{2}+\left|w-z_{k_{0}}\right|^{2} \leq\left|z_{k_{0}}\right|^{2}$, i.e. $w \in \overline{D\left(\frac{z_{k_{0}}}{2}, \frac{\left|z_{k_{0}}\right|}{2}\right)}$.

Theorem 4. If all the zeros of a polynomial $p(z) \in C[z]$ are $z_{k}, k=1,2, \ldots, n$. Then for each zero $w$ of the derivative $p^{\prime}(z)$ exists some $k_{0} \in N, 1 \leq k_{0} \leq n$, such that $w \notin \overline{D\left(\frac{z_{k_{0}}}{2}, \frac{\left|z_{k_{0}}\right|}{2}\right)}$.

Proof. Let $w \in C$ be such that $p^{\prime}(w)=0$. We except the trivial case $p(w)=0$, which confirms the assertion.

Further we repeat the proof of Theorem 3 and we get $\alpha_{1}+\alpha_{2}+\cdots+\alpha_{n}=-1$. Consequently, there exists $k_{0} \in N, \quad 1 \leq k_{0} \leq n$ such that $\alpha_{k_{0}} \geq-1$. It means that $|w|^{2}+\left|w-z_{k_{0}}\right|^{2} \geq\left|z_{k_{0}}\right|^{2}$, i.e. $w \notin \overline{D\left(\frac{z_{k_{0}}}{2}, \frac{\left|z_{k_{0}}\right|}{2}\right)}$. 
Theorem 5. If all the zeros of the polynomial $p(z) \in C[z]$ are $z_{k}, k=1,2, \ldots, n$. Then for each zero $t$ of the second derivative $p^{\prime \prime}(z)$ exists some, $k_{0} \in N, 1 \leq k_{0} \leq n$, such that $t \in \overline{C\left(\frac{z_{k_{0}}}{2}, \frac{\left|z_{k_{0}}\right|}{2}\right)}$.

Proof. We denote by $t$ the zero of the second derivative, i.e. $p^{\prime \prime}(t)=0$.

According to Theorem 3 there exists such a zero $w$ of the derivative $p^{\prime}(z)$, that $t \in$ $\overline{D\left(\frac{w}{2}, \frac{|w|}{2}\right)}$. For this zero $w$ of the derivative $p^{\prime}(z)$, according to Theorem 3 , there exists a zero of the polynomial $p(z)-z_{k_{0}}$, such that $w \in \overline{D\left(\frac{z_{k_{0}}}{2}, \frac{\left|z_{k_{0}}\right|}{2}\right)}$.

In order to find the geometric places of the points $t$, let us take $w$ on the boundary of the disk $\overline{D\left(\frac{z_{k_{0}}}{2}, \frac{\left|z_{k_{0}}\right|}{2}\right)}$ and $t$ on the boundary of the disk $\overline{D\left(\frac{w}{2}, \frac{|w|}{2}\right)}$. For better understanding, let us take $z_{k_{0}} \in X$, and $\arg w=\alpha$, arg $t=\alpha+\beta$.

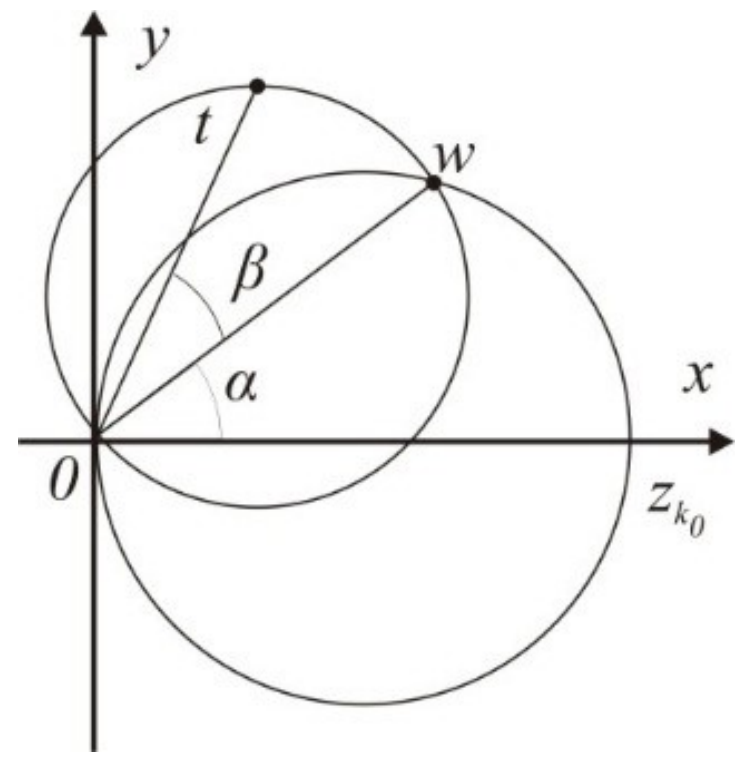

Figure 2:

Here we have $\alpha, \beta \in\left[0, \frac{\pi}{2}\right]$. Let us put $\left|z_{k_{0}}\right|=a$. Then

$$
\begin{aligned}
x & =a \cos \alpha \cos \beta \cos (\alpha+\beta), \\
y & =a \cos \alpha \cos \beta \sin (\alpha+\beta), \\
x^{2}+y^{2} & =a^{2} \cos ^{2} \alpha \cos ^{2} \beta, \\
\left(x^{2}+y^{2}-a x\right)^{2} & =\left(a^{2} \cos ^{2} \alpha \cos ^{2} \beta-a^{2} \cos \alpha \cos \beta \cos (\alpha+\beta)\right)^{2} \\
& =a^{4} \cos ^{2} \alpha \cos ^{2} \beta \sin ^{2} \alpha \sin ^{2} \beta \leq a^{2} a^{2} \cos ^{2} \alpha \cos ^{2} \beta=a^{2}\left(x^{2}+y^{2}\right) .
\end{aligned}
$$

This means that our geometric place belongs to $\overline{C\left(\frac{z_{k_{0}}}{2}, \frac{\left|z_{k_{0}}\right|}{2}\right)}$. 


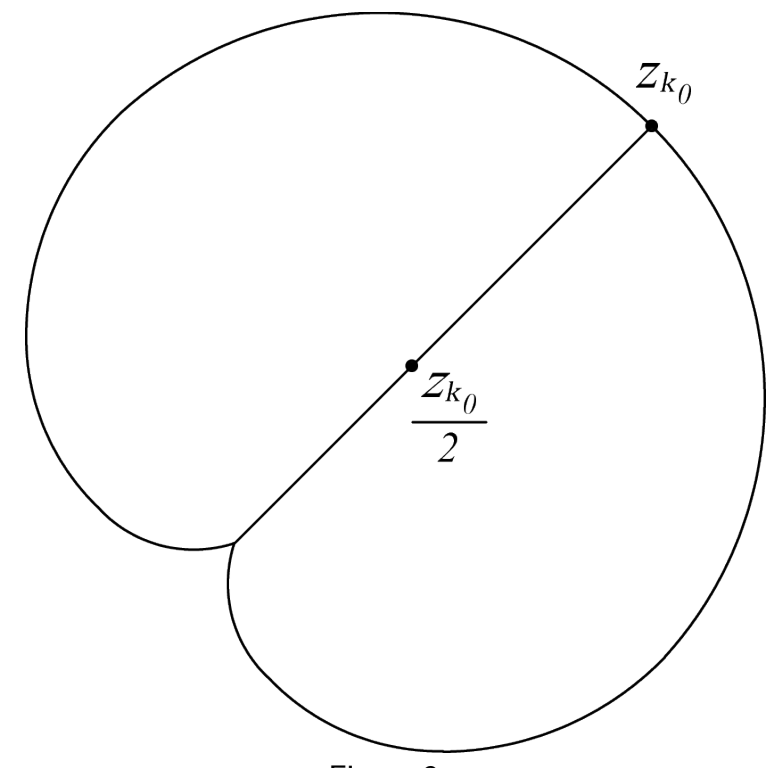

Figure 3:

\section{Main results}

Lemma 1. If $x, y \in[0,1]$, then the function $h(x, y)=x \sqrt{1-y^{2}}+y \sqrt{1-x^{2}} \leq 1$.

Proof. If we fix $y \in[0,1]$, then

$$
\begin{gathered}
t(x)=h(x, y)=x \sqrt{1-y^{2}}+y \sqrt{1-x^{2}} . \\
\frac{\partial t}{\partial x}=\sqrt{1-y^{2}}-\frac{x y}{\sqrt{1-x^{2}}}=\frac{\sqrt{1-x^{2}-y^{2}+x^{2} y^{2}}-x y}{\sqrt{1-x^{2}}},
\end{gathered}
$$

where $x \neq 1$.

The function $l(x)=\sqrt{1-x^{2}-y^{2}+x^{2} y^{2}}-x y=0$, when $1-x^{2}-y^{2}=0 \Longleftrightarrow x=$ $\sqrt{1-y^{2}}$. We know

$$
\begin{aligned}
& l(x)>0 \text { with } x<\sqrt{1-y^{2}}, \\
& l(x)<0 \text { with } x>\sqrt{1-y^{2}}, \\
& l(0)=\sqrt{1-y^{2}}>0, \\
& l(1)=-y<0 .
\end{aligned}
$$

Then $h(x, y) \leq t\left(\sqrt{1-y^{2}}\right)=1-y^{2}+y^{2}=1$, which confirms the lemma.

Theorem 6. Let $x, y, z \in[0,1]$. Then the function $f(x, y, z)=x y \sqrt{1-z^{2}}+y z \sqrt{1-x^{2}}+$ $z x \sqrt{1-y^{2}}$ satisfies $f(x, y, z) \leq \frac{2 \sqrt{3}}{3}$. 
Proof. Let us calculate

$$
\begin{aligned}
& \frac{\partial f}{\partial x}=y \sqrt{1-z^{2}}+z \sqrt{1-y^{2}}-\frac{x y z}{\sqrt{1-x^{2}}}, \\
& \frac{\partial f}{\partial y}=x \sqrt{1-z^{2}}+z \sqrt{1-x^{2}}-\frac{x y z}{\sqrt{1-y^{2}}}, \\
& \frac{\partial f}{\partial z}=x \sqrt{1-y^{2}}+y \sqrt{1-x^{2}}-\frac{x y z}{\sqrt{1-z^{2}}} .
\end{aligned}
$$

We want $x \neq 1, y \neq 1, z \neq 1$. We need to solve the system

$$
\begin{aligned}
& y \sqrt{1-z^{2}}+z \sqrt{1-y^{2}}-\frac{x y z}{\sqrt{1-x^{2}}}=0, \\
& x \sqrt{1-z^{2}}+z \sqrt{1-x^{2}}-\frac{x y z}{\sqrt{1-y^{2}}}=0, \\
& y \sqrt{1-x^{2}}+x \sqrt{1-y^{2}}-\frac{x y z}{\sqrt{1-z^{2}}}=0 .
\end{aligned}
$$

If $x \neq 0, y \neq 0, z \neq 0$, the system is

$$
\begin{aligned}
& \frac{\sqrt{1-z^{2}}}{z}+\frac{\sqrt{1-y^{2}}}{y}=\frac{x}{\sqrt{1-x^{2}}}, \\
& \frac{\sqrt{1-z^{2}}}{z}+\frac{\sqrt{1-x^{2}}}{x}=\frac{y}{\sqrt{1-y^{2}}}, \\
& \frac{\sqrt{1-x^{2}}}{x}+\frac{\sqrt{1-y^{2}}}{y}=\frac{z}{\sqrt{1-z^{2}}} .
\end{aligned}
$$

We put

$$
\frac{\sqrt{1-x^{2}}}{x}=p, \frac{\sqrt{1-y^{2}}}{y}=q, \frac{\sqrt{1-z^{2}}}{z}=s .
$$

Then we have

$$
\begin{aligned}
& q+s=\frac{1}{p}, \\
& s+p=\frac{1}{q}, \\
& p+q=\frac{1}{s} .
\end{aligned}
$$

After the subtraction $(2)-(3)$, we get $q+\frac{1}{q}=p+\frac{1}{p}$, i.e. $p=q$ or $p=\frac{1}{q}$. 
Analogously $q=s$ or $q=\frac{1}{s}$, and $s=p$ or $s=\frac{1}{p}$. i.e.

Because of (1), the only possibility is $p=q=s$ and therefore $p+p=\frac{1}{p}$, i.e. $p=\sqrt{\frac{1}{2}}$,

$$
x=y=z=\sqrt{\frac{2}{3}} .
$$

The critical point is $M\left(\sqrt{\frac{2}{3}}, \sqrt{\frac{2}{3}}, \sqrt{\frac{2}{3}}\right)$.

We have:

$$
\begin{gathered}
\frac{\partial^{2} f}{\partial x^{2}}=\frac{-y z}{\left(1-x^{2}\right)^{\frac{3}{2}}}, \quad \frac{\partial^{2} f}{\partial y^{2}}=\frac{-z x}{\left(1-y^{2}\right)^{\frac{3}{2}}}, \quad \frac{\partial^{2} f}{\partial z^{2}}=\frac{-x y}{\left(1-z^{2}\right)^{\frac{3}{2}}} \\
\frac{\partial^{2} f}{\partial x \partial y}=\sqrt{1-z^{2}}-\frac{z y}{\sqrt{1-y^{2}}}-\frac{z x}{\sqrt{1-x^{2}}}, \\
\frac{\partial^{2} f}{\partial y \partial z}=\sqrt{1-x^{2}}-\frac{x y}{\sqrt{1-y^{2}}}-\frac{x z}{\sqrt{1-z^{2}}}, \\
\frac{\partial^{2} f}{\partial z \partial x}=\sqrt{1-y^{2}}-\frac{y x}{\sqrt{1-x^{2}}}-\frac{z y}{\sqrt{1-z^{2}}} .
\end{gathered}
$$

Then for the matrix

$$
H=\left(\begin{array}{ccc}
\frac{\partial^{2} f}{\partial x^{2}} & \frac{\partial^{2} f}{\partial x \partial y} & \frac{\partial^{2} f}{\partial x \partial z} \\
\frac{\partial^{2} f}{\partial y \partial x} & \frac{\partial^{2} f}{\partial y^{2}} & \frac{\partial^{2} f}{\partial y \partial z} \\
\frac{\partial^{2} f}{\partial z \partial x} & \frac{\partial^{2} f}{\partial z \partial y} & \frac{\partial^{2} f}{\partial z^{2}}
\end{array}\right)
$$

we get

$$
H(M)=\left(\begin{array}{ccc}
-2 \sqrt{3} & -\sqrt{3} & -\sqrt{3} \\
-\sqrt{3} & -2 \sqrt{3} & -\sqrt{3} \\
-\sqrt{3} & -\sqrt{3} & -2 \sqrt{3}
\end{array}\right)=+\sqrt{3}\left(\begin{array}{ccc}
-2 & -1 & -1 \\
-1 & -2 & -1 \\
-1 & -1 & -2
\end{array}\right)
$$

The determinants:

$$
D_{1}=|-2|=-2<0, D_{2}=\left|\begin{array}{ll}
-2 & -1 \\
-1 & -2
\end{array}\right|=3>0, D_{3}=\left|\begin{array}{ccc}
-2 & -1 & -1 \\
-1 & -2 & -1 \\
-1 & -1 & -2
\end{array}\right|=-4<0 \text {. }
$$

That means: $M$ is a local maximum $f(M)=\frac{2 \sqrt{3}}{3}$. On the plane $z=0$ we have $r(x, y)=$ $f(x, y, 0)=x y \leq 1$. On the plane $z=1$ we have $h(x, y)=f(x, y, 1)=x \sqrt{1-y^{2}}+$ $y \sqrt{1-x^{2}} \leq 1$, according to the Lemma 1 . Because $f$ is a symmetric function and defined in the cube $[0,1]^{3}$, therefore $f \leq \frac{2 \sqrt{3}}{3}$. 
Corollary 1. If $\alpha, \beta, \gamma \in\left[0, \frac{\pi}{2}\right]$, then the function

$g(\alpha, \beta, \gamma)=\sin \alpha \sin \beta \cos \gamma+\sin \alpha \sin \gamma \cos \beta+\sin \beta \sin \gamma \cos \alpha \leq \frac{2 \sqrt{3}}{3}$.

Proof. If in the conditions of Theorem 6 we put $x=\sin \alpha, y=\sin \beta, z=\sin \gamma$, and we obtain that

$$
g(\alpha, \beta, \gamma)=f(x, y, z) \leq \frac{2 \sqrt{3}}{3}
$$

Theorem 7. If all the zeros of the polynomial $p(z) \in C[z]$ are $z_{k}, k=1,2, \ldots, n$. Then for each zero $v$ of the third derivative $p^{\prime \prime \prime}(z)$ exists some $k_{0} \in N, \quad 1 \leq k_{0} \leq n$, such that $v \in \overline{C_{q}\left(\frac{z_{k_{0}}}{2}, \frac{\left|z_{k_{0}}\right|}{2}\right)}$, where $q=\frac{2 \sqrt{3}}{3}$.

Proof. We denote by $v$ zero of the third derivative, i.e. $p^{\prime \prime \prime}(v)=0$.

According to Theorem 3 there exists such a zero $t$ of the second derivative $p^{\prime \prime}(z)$, that $v \in \overline{D\left(\frac{t}{2}, \frac{|t|}{2}\right)}$. For this zero $t$ according again to Theorem 3 , there exists a zero $w$ of the polynomial $p^{\prime}(z)$, such that $t \in \overline{D\left(\frac{w}{2}, \frac{|w|}{2}\right)}$.

And again for this zero $w$ of the derivative $p^{\prime}(z)$, there exists a zero $z_{k_{0}}$ of the polynomial $p(z)$, such that $w \in \overline{D\left(\frac{z_{k_{0}}}{2}, \frac{\left|z_{k_{0}}\right|}{2}\right)}$.

In order to find the geometric places of the points $t$, let us take $w$ on the boundary of the disk $\overline{D\left(\frac{z_{k_{0}}}{2}, \frac{\left|z_{k_{0}}\right|}{2}\right)}$ and $t$ on the boundary of the disk $\overline{D\left(\frac{w}{2}, \frac{|w|}{2}\right)}$. For better understanding, let us take $z_{k_{0}} \in X$, and $\arg w=\alpha$, arg $t=\alpha+\beta$, arg $v=\alpha+\beta+\gamma$.

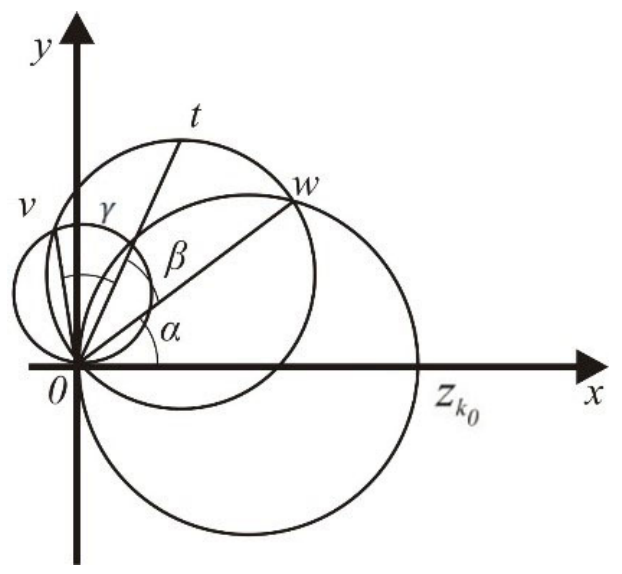

Figure 4:

Here we have $\alpha, \beta, \gamma \in\left[0, \frac{\pi}{2}\right]$. 
Let us put $\left|z_{k_{0}}\right|=a$. Then

$$
\begin{aligned}
& x=a \cos \alpha \cos \beta \cos \gamma \cos (\alpha+\beta+\gamma), \\
& y=a \cos \alpha \cos \beta \cos \gamma \sin (\alpha+\beta+\gamma) .
\end{aligned}
$$

Let us calculate

$$
\begin{gathered}
a x=a^{2} \cos \alpha \cos \beta \cos \gamma[\cos (\alpha+\beta) \cos \gamma-\sin (\alpha+\beta) \sin \gamma] \\
=a^{2} \cos \alpha \cos \beta \cos \gamma[\cos \alpha \cos \beta \cos \gamma-\sin \alpha \sin \beta \cos \gamma \\
-\sin \alpha \sin \gamma \cos \beta-\sin \beta \sin \gamma \cos \alpha] .
\end{gathered}
$$

Obviously

$$
x^{2}+y^{2}=a^{2} \cos ^{2} \alpha \cos ^{2} \beta \cos ^{2} \gamma
$$

Then we have

$$
\begin{aligned}
x^{2}+y^{2}-a x= & a^{2} \cos \alpha \cos \beta \cos \gamma[\sin \alpha \sin \beta \cos \gamma+\sin \alpha \sin \gamma \cos \beta \\
& +\sin \beta \sin \gamma \cos \alpha] .
\end{aligned}
$$

But

$$
\sin \alpha \sin \beta \cos \gamma+\sin \alpha \sin \gamma \cos \beta+\sin \beta \sin \gamma \cos \alpha \leq \frac{2 \sqrt{3}}{3},
$$

according to the Corollary.

From here we state

$$
\left(x^{2}+y^{2}-a x\right)^{2} \leq \frac{4}{3} a^{2}\left(x^{2}+y^{2}\right) .
$$

That means that our geometric place belongs to $\overline{C_{\frac{2 \sqrt{3}}{3}}\left(\frac{z_{k_{0}}}{2}, \frac{\left|z_{k_{0}}\right|}{2}\right)}$, which confirms this assertion.

\section{Acknowledgements}

This research was financed from University of Economics of Varna research grants No.19 2018-04-27.

\section{References}

[1] Q I Rahman and G Schmeisser. Analytic Theory of Polynomials: Critical Points, Zeros and Extremal Properties. Clarendon Press, London, 2002.

[2] D M Souroujon and T Zapryanova. On the relation between the number of real and complex zeros of polynomials of a certain kind. In AIP Conference Proceeding., volume 2159, St Constantine and Helena, 2019. Sixth International Conference on New Trends in the Applications of Differential Equations in Sciences. 
[3] T Stoyanov. About the Zeros of Some Entire Functions and Their Derivatives. Journal of the Australian Mathematical Society, 68:165-169, 2000.

[4] T Stoyanov. Some Localization of the Zeros of the Derivatives of a Complex Polynomial in the Disks or Cardioid Interiorities. International Journal of Mathematical Analysis, 9(58):2849-2855, 2015.

[5] T Zapryanova. Best approximation and Moduli of Smoothness. Pliska Studia Mathematica Bulgarica, 21(1):299-306, 2012.

[6] T Zapryanova and D Souroujon. On the Iterates of Jackson Type Operator $G_{s, n}$. Mediterr. J. Math, 13:5053-5061, 2016. 